\section{Severe pulmonary vascular change in patients dying with right ventricular failure after heart transplantation}

\author{
P S Hasleton, N H Brooks
}

\begin{abstract}
Two patients died following heart transplantation from failure of the donor right ventricle. Histological examination of the lungs showed occlusion of pulmonary veins. A more reliable method is required to predict the outcome for potential heart transplant recipients with a "borderline" pulmonary vascular resistance.

(Thorax 1995;50:210-212)
\end{abstract}

Keywords: heart transplantation, outcome, pulmonary vascular resistance.

Pulmonary veno-occlusive disease (PVOD) is an important cause of pulmonary arterial hypertension with a range of aetiologies. ${ }^{1}$ We report two patients with occlusion of pulmonary veins who died following heart transplantation from failure of the donor right ventricle. Preoperative measurements had pointed to an increased pulmonary vascular resistance but were not considered to preclude successful transplantation.

\section{Case reports \\ CASE 1}

A 46 year old woman with a two year history of congestive cardiomyopathy was transferred to Wythenshawe Hospital for emergency heart transplantation. She was hypotensive and in severe congestive heart failure. Her echocardiogram showed dilated hypocontractile ventricles.

Pulmonary artery pressure (PAP) was 52/ $25 \mathrm{~mm} \mathrm{Hg}$ (mean $34 \mathrm{~mm} \mathrm{Hg}$ ), pulmonary artery wedge (PAW) mean pressure $24 \mathrm{~mm}$ $\mathrm{Hg}$, and right atrial mean pressure (RAP) $20 \mathrm{~mm} \mathrm{Hg}$. Her cardiac output, measured by the Fick method utilising assumed oxygen uptake, was 2.5 litres/minute, and the pulmonary vascular resistance (PVR) was 4 units.

The following day orthotopic heart transplantation was carried out. When weaned from cardiopulmonary bypass she was hypotensive despite inotropic support and the right ventricle was distended. Her condition deteriorated during the first two hours and she was returned to theatre. Mean PAP pressure was $30 \mathrm{~mm} \mathrm{Hg}$, systemic arterial mean pressure $50 \mathrm{~mm} \mathrm{Hg}$, central venous pressure $16 \mathrm{~mm} \mathrm{Hg}$, and the left atrial pressure $6 \mathrm{~mm} \mathrm{Hg}$. Her condition deteriorated despite prostacyclin and she died one hour later.

The excised heart weighed $380 \mathrm{~g}$. All the chambers were dilated. The ventricular weights ${ }^{2}$ were right, $86 \mathrm{~g}$ (upper limit of normal, $65 \mathrm{~g}$ ) and left, $335 \mathrm{~g}$ (upper limit of normal, $190 \mathrm{~g})$.

The donor heart weighed $455 \mathrm{~g}$. The right atrium and ventricle $(82 \mathrm{~g})$ were dilated. The left ventricle was at the upper limit of normal weight at $190 \mathrm{~g}$. Subendocardial haemorrhage was seen in the left ventricle. The coronary arteries were normal. The lungs (the histology of which is described below) showed pulmonary oedema.

CASE 2

A 38 year old man developed severe congestive heart failure associated with mitral incompetence two months after his second myocardial infarction. Angiography showed grossly impaired myocardial function (ejection fraction $10 \%$ ), severe mitral regurgitation, and diffuse disease affecting all main coronary branches.

The mean PAP was $38 \mathrm{~mm} \mathrm{Hg}$, mean PAW $19 \mathrm{~mm} \mathrm{Hg}$, cardiac output (by thermal dilution) $4 \cdot 31 / \mathrm{min}$, and pulmonary vascular resistance 4.4 units. The pulmonary vascular resistance corrected for body surface area was $7 \cdot 5$ units $/ \mathrm{m}^{2}$.

Orthotopic heart transplantation was carried out. He was returned to intensive care requiring pacing, intravenous dobutamine and adrenaline, and intra-aortic balloon pumping. Soon afterwards his heart went into ventricular fibrillation. After DC cardioversion his chest was reopened and the right ventricle was found to be distended. Mean systemic arterial pressure was $60 \mathrm{~mm} \mathrm{Hg}$, and mean PAP $30 \mathrm{~mm} \mathrm{Hg}$, despite a left atrial pressure of $8 \mathrm{~mm} \mathrm{Hg}$. Cardiopulmonary bypass was recommenced but he died.

The excised heart weighed $389 \mathrm{~g}$, right ventricular weight $81 \mathrm{~g}$, and the left $260 \mathrm{~g}$. The donor heart weighed $425 \mathrm{~g}$. The right atrium and ventricle $(59 \mathrm{~g})$ were dilated. The left weighed $150 \mathrm{~g}$.

\section{HISTOLOGICAL EXAMINATION}

Histological examination of the lungs showed intra-alveolar haemorrhage and histiocytes in case 2. Recent thrombus with some organisation was seen in large muscular pulmonary arteries of the left lower lobe in case 1. There was interstitial oedema and some margination of neutrophil polymorphs.

Both cases showed occlusion of pulmonary veins by intimal fibrosis (fig 1) and muscularisation of the pulmonary veins with a distinct media between an internal and external lamina. Intimal longitudinal muscle was common. There was evidence of pulmonary arterial hypertension with hypertrophy of muscular pulmonary arteries (fig 2) and muscularisation of pulmonary arterioles. No fibrinoid necrosis or plexiform lesions were seen. 


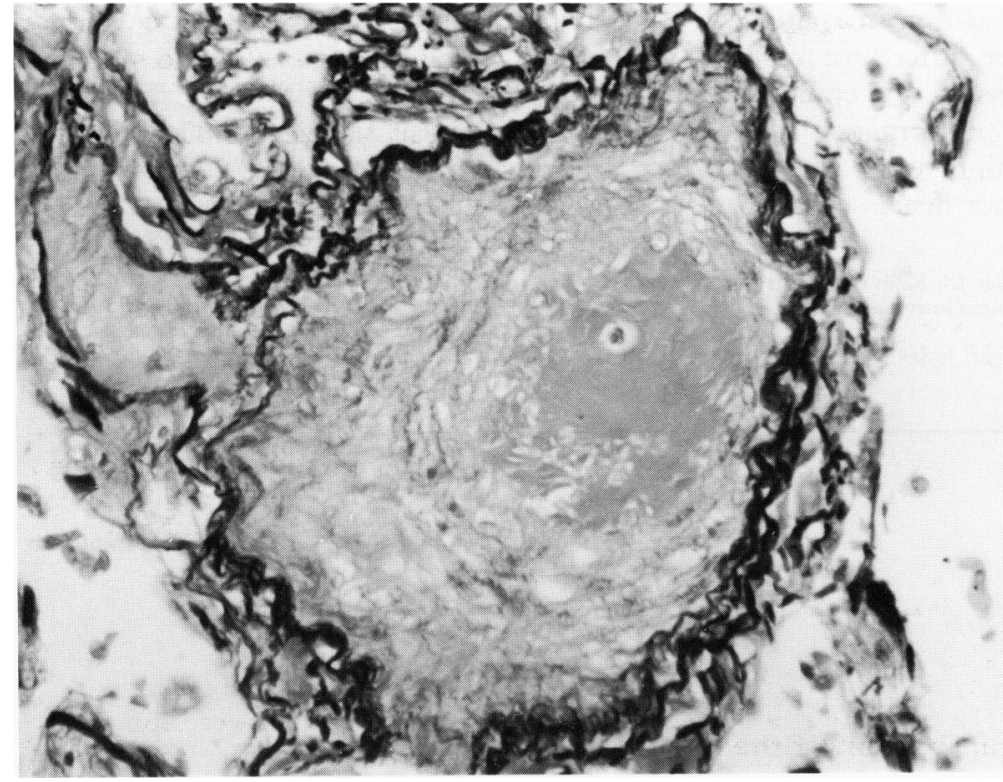

Figure 1 Pulmonary vein occluded by intimal fibrosis with evidence of recanalisation. Stain: elastic van Geison, magnification $\times 313$ reduced to $69 \%$ in origination.

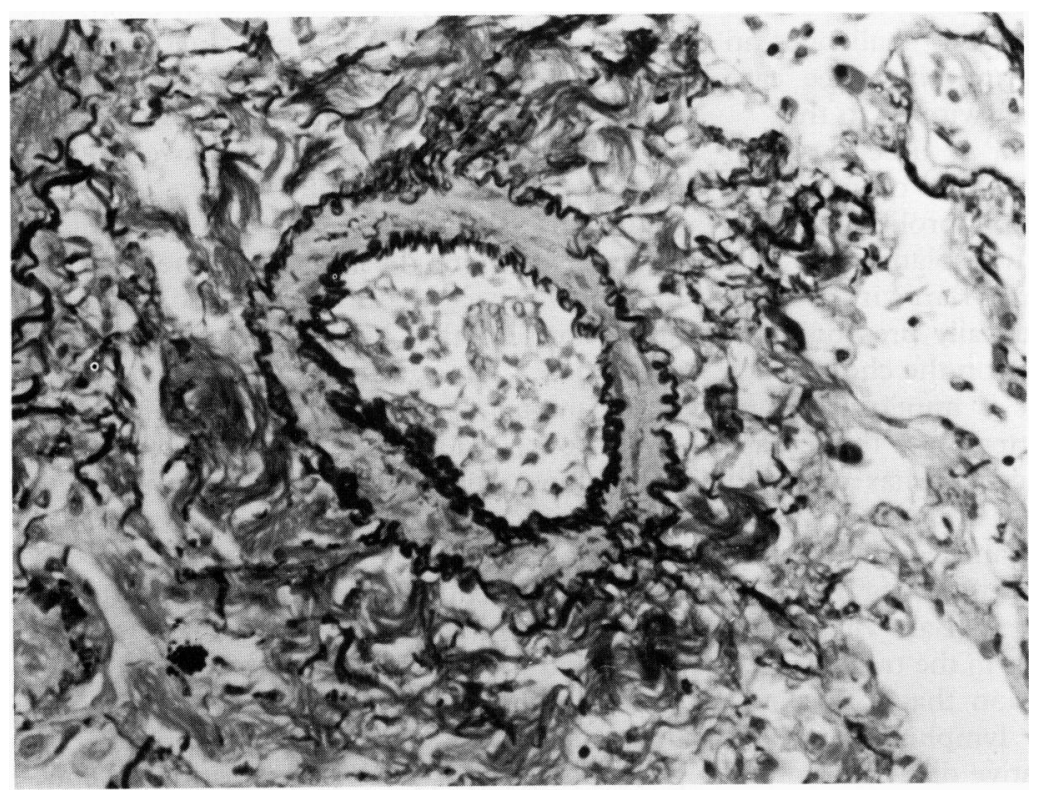

Figure 2 Hypertrophied muscular pulmonary artery. Stain: elastic van Geison, magnification $\times 313$ reduced to $69 \%$ in origination.

\section{Discussion}

Irreversible pulmonary hypertension causing failure of the donor right ventricle is an important cause of death after heart transplantation, and all potential recipients undergo right heart catheterisation as part of their assessment. The risk increases progressively with measures of elevated pulmonary resistance, ${ }^{3}$ but no agreement exists on the most predictive criteria or the level at which a patient should be considered inoperable. The so-called pulmonary vascular resistance - that is, the pulmonary pressure-flow rates - possibly corrected for body surface area, should be a reliable index, ${ }^{3}$ but in one study the transpulmonary gradient proved to be a better predictor of risk, ${ }^{4}$ perhaps because of the inaccuracy inherent in clinical measurement of cardiac output.

We were alerted to the potential for right ventricular failure in both our patients: in case
1 by the high systolic pulmonary artery pressure and the transpulmonary gradient of $15 \mathrm{~mm} \mathrm{Hg}$ measured a year earlier, and in case 2 by the transpulmonary gradient of $19 \mathrm{~mm} \mathrm{Hg}$ and the pulmonary vascular resistance index of 7.5 units $/ \mathrm{m}^{2}$; both patients had right axis deviation on the electrocardiogram and case 1 had criteria for right atrial enlargement. Preoperative right heart catheterisation in case 1 , however, appeared to be satisfactory with a transpulmonary gradient of $10 \mathrm{~mm} \mathrm{Hg}$, and we had not anticipated advanced pulmonary vascular disease in case 2 since his deterioration had occurred over two weeks. He may have had severe asymptomatic mitral regurgitation since his first myocardial infarction four years earlier. He was too ill for an assessment to be made of the response of his pulmonary resistance to vasodilatation.

Occlusion of pulmonary veins might have resulted in underestimation of the pulmonary vascular resistance by elevating the pulmonary artery wedge pressure above the left atrial pressure. Unfortunately the opportunity to test this possibility by direct comparison of the pulmonary artery and left atrial pressures at operation was missed, but disproportionate elevation of the pulmonary artery wedge pressure would, according to theoretical considerations, be expected only with obliteration of large pulmonary veins. ${ }^{5}$

The pulmonary changes causing pulmonary hypertension were due to pulmonary venous occlusion secondary to left ventricular failure. These changes consisted of muscularised pulmonary arterioles, hypertrophied muscular pulmonary arteries, intimal fibrosis and intimal longitudal muscle in pulmonary veins, as well as muscularisation of veins. In neither case was there evidence of any other cause of raised left atrial pressure. In addition to these structural changes it is likely that the pulmonary resistance was further increased by raised complement levels resulting from cardiothoracic surgery. ${ }^{6} \mathrm{It}$ is most probable, however, that the morphological changes were most important in causing the pulmonary arterial and venous hypertension, which was unaffected by the infusions of prostacyclin.

The unpredictable outcome of heart transplantation in patients with borderline pulmonary haemodynamics is likely to be determined by donor factors including size, the use of inotropic agents prior to death, the ischaemic time, and the presence of unrecognised coronary or myocardial disease. An additional factor is the imprecision of right heart catheterisation. Histological examination of the lungs of potential recipients might provide a better indication of the severiry of underlying pulmonary vascular disease. We are investigating this possibility with a prospective correlation of preoperative measurements of pulmonary artery pressure and resistance with clinical outcome and morphological examination of the lung biopsy sample taken at the time of transplantation, though we recognise that routine assessment of lung histology in potential heart transplant recipients might be precluded by the risk of an operation 
to obtain an adequate biopsy sample in a patient with severe heart failure. The procedure might be justified in borderline cases, if it could be relied upon to avoid the performance of a hopeless transplant operation with the attendant loss of a scarce donor heart.

1 Hasleton PS, Ironside JW, Whittaker JS, Kelly W, Ward C, Thompson GS. Pulmonary veno-occlusive disease. Histopathology 1986;10:933-44.

2 Fulton RM, Hutchinson EC, Morgan Jones M. Ventricula weight in cardiac hypertrophy. Br Heart f 1952;14:413-20. 3 Kirklin JK, Naftal DC, Kirklin JW, Blackstone EH, WhiteWilliams C, Bourge RC. Pulmonary vascular resistance and the risk of heart transplantation. $f$ Heart Transplant 1988;7:331-6.

4 Kormos RL, Thompson M, Hardesty RL, Griffiths BP, Trento A, Uretsky BS, et al. Utility of preoperative right heart catheterisation data as a predictor of survival after
heart transplantation. $₹$ Heart Transplant $1986 ; 5: 391$.

5 Harris $\mathrm{P}$, Heath $\mathrm{D}$. The human pulmonary circulation. It form and function in health and disease. Edinburgh: Churchill Livingstone, 1986.

6 Tenneberg SD, Clardy CW, Bailey WW, Solomkin JS. Complement activation and lung permeability of cardiac pulmonary bypass. Ann Thorac Surg 1990;50:597-601.

\section{Commentary}

\section{Paul Corris}

Thoracic allograft transplantation has offered the respiratory physician new challenges in diagnosis and treatment and the three case reports presented here bear witness to this.

The paper by Egan et al describes two patients who developed necrotic bronchitis as a presenting feature of lymphoproliferative disease following heart-lung transplantation. This condition arises via Epstein-Barr virus driven cell transformation and usually presents with malaise, fever, and nodules on the chest radiograph. An early diagnosis is imperative since the prognosis is much better when the disease is limited in extent. The incidence has increased since the widespread use of cyclosporin as the principal immunosuppressant leading to a reduced efficacy of $T$ cell surveillance of $B$ lymphocytes infected with Epstein-Barr virus. The disease commonly presents in the transplanted organ leading to speculation that it is clonal proliferation of donor B lymphocytes which results in lymphoproliferative disease.

The importance of this paper is that it supports the idea that donor bronchus associated lymphoid tissue provided the environment for clonal proliferation of transformed B cells. It is also of particular interest for those involved in the postoperative management of lung transplant recipients, serving to remind us that an abnormal inflamed airway following transplantation does not necessarily imply vascular injury with necrosis or primary aspergillus infection. It underlines the value of performing a biopsy on available tissue when faced with a clinical problem of unknown cause.

The paper by Sheerin et al describes another rare case of airway disease associated with transplantation but in this case provided the indication for single lung transplantation. $\mathrm{Ob}$ literative bronchiolitis is a pathological entity which is a recognised sequel of various insults and conditions including viruses, toxic gas, rheumatoid arthritis, and following bone marrow or lung transplantation. It is often diagnosed after other more common diseases of airflow obstruction have been excluded. We have transplanted four patients with obliterative bronchiolitis, one following bone marrow transplantation, one associated with rheumatoid arthritis, and two of unknown aetiology. Histological examination of resected native lungs in both the latter cases confirmed the diagnosis but did not shed light on the aetiology. It is of interest, therefore, that in this case the proposed mechanism was identified by histological examination of the lung removed at transplantation. The diagnosis could have been made preoperatively if a lung biopsy had been carried out, and raises questions as to whether lung biopsy should have been considered in this patient at some stage during her 10 year history of progressive airflow obstruction. It is only by application of modern techniques of immuno and molecular pathology to lung tissue that the mechanisms behind obliterative bronchiolitis of unknown aetiology will be determined, hopefully leading to new appoaches in therapy. It is interesting to speculate whether treatment with somatostatin or its analogue octreotide would have had any effect on tumour size or degree of obstruction in this case.

The necessity of full and careful investigation of patients undergoing any form of transplantation is borne out by the paper by Hasleton and Brooks which describes two patients who died of right heart failure after cardiac transplantation. Deaths were due to pulmonary veno-occlusive disease leading to a high pulmonary vascular resistance. It was interesting to note the morphological changes in pulmonary veins associated with the raised left atrial pressure. Many transplant groups would have been very wary of transplanting patients with a pulmonary vascular resistance of 4 or more units and transpulmonary gradients of 15 and $19 \mathrm{~mm} \mathrm{Hg}$ respectively, particularly with no attempts to reduce this pharmacologically. Certainly it confirms the much greater mortality associated with heart transplantation in patients with raised pulmonary vascular resistance of whatever cause.
Unit, Montreal General Hospital, Montreal, Quebec, Canada, H3G 1A4 $P$ Corris 